\title{
Typing of emm77 Streptococcus pyogenes strains isolated from different clinical samples
}

\author{
Claudio Palmieri', Maria Stella Princivalli', Lucilla Baldassarri', Roberta Creti $^{2}$, \\ Pietro Emanuele Varaldo', Bruna Facinelli' \\ 'Istituto di Microbiologia e Scienze Biomediche, Università Politecnica delle Marche, Ancona; \\ ${ }^{2}$ Dipartimento di Malattie Infettive, Parassitarie ed Immunomediate, Istituto Superiore di Sanità, Roma
}

Key words: Streptococcus pyogenes, emm77, ST63/369, erm(A) tet(O), MLST

Tipizzazione di ceppi di Streptococcus pyogenes emm77 isolati da differenti campioni clinici

\section{SUMMARY}

Population structure analysis of emm77 Streptococcus pyogenes isolates demonstrated that the Clonal Complex ST63/369, previously reported in Italian pharyngeal strains, can also be found in non-pharyngeal strains. The success of ST63/369 may be due to the association of specific resistance [iMLS-B/erm $(A)$; tet $(O)$ ] and virulence [internalization-gene prtFI] traits.

Received May 6, 2008

Accepted July 24, 2008

\section{INTRODUZIONE}

Da uno studio su ceppi di Streptococcus pyogenes isolati in Italia nel biennio 1997-98 da casi di faringo-tonsillite acuta in età pediatrica (13), è emersa una associazione inaspettata tra eritromicino-resistenza e capacità di invadere cellule epiteliali respiratorie (3). S. pyogenes è un patogeno intracellulare, in grado di invadere cellule epiteliali umane (1) e l'invasione è mediata da una sottoclasse di adesine che prendono il nome di invasine. Tra queste, un ruolo cruciale è giocato da F1, una proteina fibronectin ( $f n$ )-binding, codificata dal gene prtF1 (7). La regione ripetuta $R D 2$ di prtF1, fondamentale per il legame alla $f n$, può essere utilizzata nella tipizzazione molecolare dei ceppi (11). Il confronto dei risultati di RD2typing, emm-typing e PFGE con il genotipo/fenotipo di resistenza consentiva di identificare numerosi cloni resistenti ed invasivi in circolazione in Italia (11). In particolare, tre cloni rappresentavano il $40 \%$ di tutti i ceppi studiati: (i) iMLS$\mathrm{B} / \operatorname{erm}(\mathrm{A})$, emm77; (ii) $\mathrm{M} / m e f(\mathrm{~A}), e m m 4$; e (iii) iMLS-A/erm(B), emm89.

In un analogo studio di popolazione (NORM 2002), condotto su ceppi resistenti ai macrolidi isolati in Norvegia da differenti campioni clinici nel 1998-2002 e tipizzati mediante emm-typing e multilocus sequence typing (MLST) (5), è emerso che la diffusione di $S$. pyogenes in Norvegia era ugualmente policlonale e che quattro cloni com- prendevano il 75\% dei ceppi analizzati: (i) erm(A), emm77, sequence type (ST) 63/369; (ii) erm(A), emm22, ST46/368; (iii) mef(O), emmstNS1033, ST205; e (iv) mef(A), emm4, ST39. Nel 2006 uno studio comparativo, condotto su ceppi isolati in Italia da faringe e ceppi isolati da differenti campioni clinici in Norvegia, ha messo in evidenza che i ceppi erm(A) emm77 isolati in Italia e Norvegia appartengono allo stesso clone (ST63/369) (9).

In questo studio, ceppi emm77, isolati in Italia nel 2003-2005 da differenti campioni clinici, sono stati sottoposti a tipizzazione fenotipica e genotipica per verificare la persistenza sul territorio italiano di ST63/369.

\section{MATERIALE E METODI}

Ceppi. Lo studio comprendeva 10 ceppi di S. pyogenes emm77 di cui 6 isolati da faringe, 2 da sangue, 1 da ferita, ed 1 da un caso di toxic shock syndrome (TSS) (tabella 1). SP1900 (ST63/369, erm(A), emm77) (9) veniva utilizzato come controllo.

Determinazione fenotipo e genotipo di resistenza. La sensibilità a macrolidi e tetracicline è stata determinata mediante metodo di Kirby Bauer. La determinazione del fenotipo di resistenza ai macrolidi è stata eseguita mediante tecnica del triplo dischetto come descritto da Giovanetti et al. (4). La presenza di geni erm, mef, e tet è stata sag-

\section{Corresponding author: Claudio Palmieri}

Istituto di Microbiologia e Scienze Biomediche - Università Politecnica delle Marche

Via Tronto I0/A, 60100 Ancona - Italia

Tel: + 39 07I 2206292 - Fax: + 39 07I 2206293 - E-mail: unopunto26@virgilio.it 
giata mediante PCR utilizzando primers specifici, come precedentemente descritto $(8,9)$.

Tipizzazione molecolare. I ceppi sono stati tipizzati mediante studio della regione $R D 2$ di $p r t F 1$ (RD2-typing), PFGE dopo macrorestrizione del DNA genomico con SmaI ed MLST. RD2 typing è stata condotta mediante PCR e digestione degli amplificati con HaeIII come precedentemente descritto (11): il pattern di restrizione definiva il tipo RD2. La MLST è stata effettuata utilizzando 7 loci house keeping: glucose kinase (gki), glutamine transporter protein ( $g$ tr), glutamate racemase (murI), DNA mismatch repair protein (mutS), transketolase (recP), xanthine phosphoribosyl transferase (xpt), ed acetyl coenzyme A acetyltransferase (yqiL) come descritto da Enright et al. (2); il numero allelico di ciascun locus ed il ST sono stati ottenuti dal sito web http://www.mlst.net.

\section{RISULTATI}

Fenotipo e genotipo di resistenza. Ad eccezione del ceppo SP5647, resistente a tetracicline tet(M) e sensibile a macrolidi, rimanenti ceppi saggiati erano resistenti sia a tetraciclina [tet $(\mathrm{O})]$ che a macrolidi [erm(A), fenotipo inducibile (iMLS-B)] (tabella 1).

RD2 typing. In tutti i ceppi, mediante PCR si ottenevano un amplificato di 349 bp consistente con la presenza di due ripetizioni complete da 111 bp ed una incompleta da 97 bp. L'analisi di restrizione con HaeIII metteva in evidenza 2 tipi $R D 2$ (tabella 1): tipo d, presente in 9 ceppi (compreso il ceppo di controllo SP1900); e tipo x presente nel ceppo SP5647.

PFGE ed MLST. La PFGE evidenziava 4 pulsotipi (figura I), che differivano dal pulsotipo del ceppo SP1900 per 2 bande (pulsotipo A, SP4156, SP4170, SP5984, SP6040, SP6064, SP6085), per 3 bande (pulsotipo C, SP4638,

Tabella I. Caratteristiche dei ceppi di S. pyogenes emm77:fenotipo/genotipo di resistenza, RD2, PFGE e Sequence Type (ST)

\begin{tabular}{|c|c|c|c|c|c|c|}
\hline Ceppo & Origine & $\begin{array}{c}\text { Fenotipo/Genotipo di } \\
\text { resistenza } \\
\text { ai macrolidi }\end{array}$ & $\begin{array}{l}\text { Genotipo di } \\
\text { resistenza } \\
\text { alla tetraciclina }\end{array}$ & $\begin{array}{l}\text { RD2 } \\
\text { type* }\end{array}$ & $\begin{array}{c}\text { PFGE } \\
\text { type }\end{array}$ & $\begin{array}{c}\text { Sequence } \\
\text { type }\end{array}$ \\
\hline SP4I56 & Faringe & iMLS-B/erm(A) & $\operatorname{tet}(\mathrm{O})$ & d & A & ST63/369 \\
\hline SP4I70 & Faringe & iMLS-B/erm(A) & $\operatorname{tet}(\mathrm{O})$ & d & A & ST63/369 \\
\hline SP4638 & Faringe & iMLS-B/erm(A) & tet $(\mathrm{O})$ & d & $\mathrm{C}$ & ST63/369 \\
\hline SP4678 & Faringe & iMLS-B/erm(A) & tet $(\mathrm{O})$ & d & C & ST63/369 \\
\hline SP5984 & Ferita & iMLS-B/erm(A) & $\operatorname{tet}(\mathrm{O})$ & d & A & ST63/369 \\
\hline SP5II0 & TSS & iMLS-B/erm(A) & tet $(\mathrm{O})$ & d & $\mathrm{D}$ & ST63/369 \\
\hline SP5647 & Sangue & Sensibile & $\operatorname{tet}(\mathrm{M})$ & $x$ & $E$ & ST399 \\
\hline SP6040 & Faringe & iMLS-B/erm(A) & $\operatorname{tet}(\mathrm{O})$ & d & $\mathrm{A}$ & ST63/369 \\
\hline SP6064 & Faringe & iMLS-B/erm(A) & tet $(\mathrm{O})$ & d & $\mathrm{A}$ & ST63/369 \\
\hline SP6085 & Sangue & iMLS-B/erm(A) & tet(O) & d & A & ST63/369 \\
\hline SPI900 & Faringe & iMLS-B/erm(A) & tet(O) & d & $B$ & ST63/369 \\
\hline
\end{tabular}

*: d, tipo RD2 descritto da Spinaci et al. (II); $x$, nuovo

A

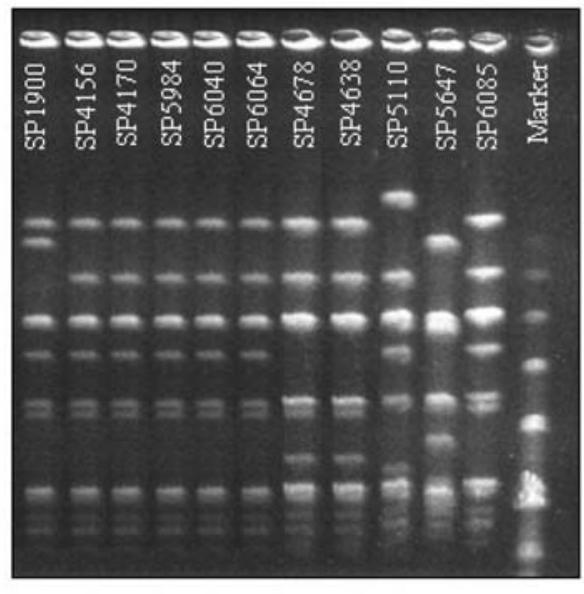

B

$\%$ similarità

80

90

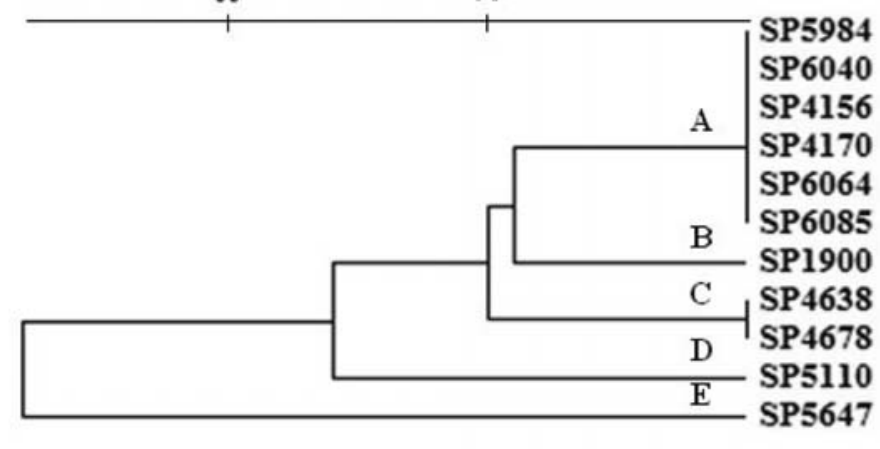

Figura I. Tipi PFGE (A) e dendrogramma (B) dei ceppi di S. pyogenes studiati. M, marker (Low Range PFG marker, New England Biolabs, Beverly, Mass) 
SP4678), per 5 bande (pulsotipo D, SP5110; pulsotipo E, SP5647). I risultati della MLST dimostravano che il ceppo SP5647 apparteneva a ST399 mentre i restanti ceppi appartenevano al complesso clonale ST63/369.

\section{DISCUSSIONE}

In base ai criteri di interpretazione dei profili di PFGE descritti da Tenover et al. (12), otto ceppi, tutti $\operatorname{erm}(\mathrm{A}) \operatorname{tet}(\mathrm{O})$ e provenienti da differenti campioni clinici (faringe, ferita, sangue), risultavano strettamente correlati a SP1900, isolato in origine da faringe; la stretta correlazione veniva confermata dai risultati della MLST, che dimostravano l'appartenenza degli isolati a ST63/369. Il ceppo erm(A) tet(O), isolato da un caso di TSS, veniva assegnato al complesso clonale ST63/369 ma tramite PFGE risultava solo possibilmente correlato al controllo.

Il ceppo SP5647, isolato da sangue, è risultato essere l'unico ceppo sensibile a macrolidi. La PFGE lo indicava come possibilmente correlato a SP1900 ma la MLST lo assegnava ad un ST differente (ST399), descritto precedentemente in Thailandia (10). RD2-typing confermava $\mathrm{i}$ risultati della MLST, in quanto tutti gli isolati ST63/369 presentavano lo stesso tipo, mentre il серро ST399 presentava un tipo RD2 mai descritto in precedenza.

I risultati di questo studio evidenziano la persistenza nel tempo di ST63/369 tra i ceppi faringei in circolazione in Italia, documentano la sua presenza anche tra ceppi non faringei e suggeriscono che eventi genetici recenti possano avere modificato il profilo di restrizione del ceppo da TSS.

Il successo di ST63/369 può derivare dal fatto che i ceppi resistenti ed invasivi presentano evidenti vantaggi selettivi: possono infatti sfuggire alla penicillina ed agli altri ß-lattamici grazie alla localizzazione intracellulare ed ai macrolidi grazie alla resistenza.

Inoltre, la presenza di ST63/369 tra ceppi di $S$. pyogenes, isolati sia nel corso di infezioni non invasive che di infezioni invasive, può essere spiegata dal particolare tipo emm (emm77), che non mostra preferenza per un particolare sito di infezione (6).

\section{BIBLIOGRAFIA}

1. Cleary PP, Cue D. High frequency invasion of mammalian cells by beta hemolytic streptococci. Subcell Biochem, 2000; 33: 137-66.

2. Enright MC, Spratt BG, Kalia A, Cross JH, Bessen DE. Multilocus sequence typing of Streptococcus pyogenes and the relationships between emm type and clone. Infect Immun, 2001; 69: 2416-27.

3. Facinelli B, Spinaci C, Magi G, Giovanetti E, Varaldo $\mathrm{PE}$. Association between erythromycin resistance and ability to enter human respiratory cells in group A streptococci. Lancet, 2001; 358: 30-3.

4. Giovanetti E, Montanari MP, Mingoia M, Varaldo PE. Phenotypes and genotypes of erythromycin-resistant Streptococcus pyogenes strains in Italy and heterogeneity of inducibly resistant strains. Antimicrob Agents Chemother 1999; 43: 1935-40.

5. Littauer P, Sangvik M, Caugant DA, Høiby EA, Simonsen GS, Sundsfjord A. Macrolide-resistant Streptococcus pyogenes in Norway: population structure and resistance determinants. Antimicrob Agents Chemother, 2006; 50: 1896-9.

6. McGregor KF, Spratt BG, Kalia A, et al. Multilocus sequence typing of Streptococcus pyogenes representing most known emm types and distinctions among subpopulation genetic structures. J Bacteriol, 2004; 186: 4285-94.

7. Molinari G, Chhatwal GS. Role played by the fibronectin-binding protein SfbI (Protein F1) of Streptococcus pyogenes in bacterial internalization by epithelial cells. J Infect Dis, 1999; 179: 1049-50.

8. Olsvik B, Olsen I, Tenover FC. Detection of tet(M) and tet $(\mathrm{O})$ using the polymerase chain reaction in bacteria isolated from patients with periodontal disease. Oral Microbiol Immunol, 1995; 10: 87-92.

9. Palmieri C, Vecchi M, Littauer P, Sundsfjord A, Varaldo PE, Facinelli B. Clonal spread of macrolideand tetracycline-resistant [erm(A) tet(O)] emm77 Streptococcus pyogenes isolates in Italy and Norway. Antimicrob Agents Chemother, 2006; 50: 4229-30.

10. Robinson DA, Sutcliffe JA, Tewodros W, Manoharan A, Bessen DE. Evolution and global dissemination of macrolide-resistant group A streptococci. Antimicrob Agents Chemother, 2006; 50: 2903-2911.

11. Spinaci C, Magi G, Zampaloni C, et al. Genetic diversity of cell-invasive erythromycin-resistant and susceptible group A streptococci determined by analysis of the $R D 2$ region of the prtF1 gene. J Clin Microbiol, 2004; 42: 639-44.

12. Tenover FC, Arbeit RD, Goering RV, et al. Interpreting chromosomal DNA restriction patterns produced by pulsed-field gel electrophoresis: criteria for bacterial strain typing. J Clin Microbiol, 1995; 33: 2233-9.

13. Varaldo PE, Debbia EA, Nicoletti G, et al. Nationwide survey in Italy of treatment of Streptococcus pyogenes pharyngitis in children: influence of macrolide resistance on clinical and microbiological outcomes. Clin Infect Dis, 1999; 29: 869-873. 